\title{
Minimum 3.5-year outcomes of operative treatment for Achilles tendon partial tears in the midportion and retrocalcaneal area
}

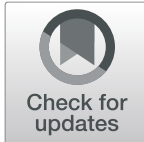

Heinz Lohrer ${ }^{1,2}$ (D)

\begin{abstract}
Background: Achilles tendon partial tears are not easy to diagnose and to manage. Most frequently, they are located in the midportion and insertional area. These entities result from different pathologic pathways, and different treatment strategies are applied. The outcome is rarely investigated.
\end{abstract}

Methods: This study includes patients who underwent surgery for partial tears in the midportion or retrocalcaneal Achilles tendon area between the years 2009 and 2015 by a single surgeon. Patients were prospectively assessed preoperatively and 3, 6, and 12 months postoperatively, using the VISA-A-G questionnaire. The final retrospective follow-up was performed after a minimum of 3.5 years postoperatively. Forty-eight Achilles tendon partial tears at the level of the retrocalcaneal bursa (impingement lesions) and 27 midportion Achilles tendon partial tears were identified. After applying rigorous exclusion criteria, 21 and 16 cases, respectively, remained for the final follow-up. Results were analysed by inferential and descriptive statistics.

Results: The VISA-A-G outcome scores improved significantly from preoperative to 6 months, 12 months, and final postoperative assessment. Preoperatively, the average VISA-A-G score was 42.1 (range, 18-73) for patients operated for Achilles tendon partial tears at the level of the retrocalcaneal bursa and 44.6 (range, 10-73) for the midportion Achilles tendon partial tear group, respectively. At final follow-up 88.8 (range, 15 to 100) and 96.9 (range, 71 to 100) were scored in the respective treatment groups. A repeated measures ANOVA determined that mean performance levels showed a statistically significant difference between measurements $(p<0.001)$. There was no systematic effect found between groups ( $p=0.836$ ).

Conclusions: In Achilles tendon partial tears recalcitrant to conservative treatment, operative intervention is highly successful in most cases, irrespective of the level of the injury. Results were statistically equal when comparing the midportion and retrocalcaneal Achilles tendon partial tear groups.

Trial registration: DRKS, DRKS00014266. Registered 06 April 2018. 'Retrospectively registered', https://www.drks.de/ drks_web/navigate.do?navigationld=results.

Keywords: Achilles tendon partial tear, VISA-A, Haglund's disease, Retrocalcaneal bursitis, Operation, Results

\footnotetext{
Correspondence: lohrer@esn-ortho.de

'European SportsCare Network (ESN), Zentrum für Sportorthopädie,

Borsigstrasse 2, 65205 Wiesbaden-Nordenstadt, Germany

${ }^{2}$ Department for Sports and Sport Science, Albert-Ludwigs-Universität

Freiburg i. Brsg., Schwarzwaldstraße 175, 79117 Freiburg, Germany
}

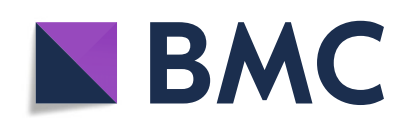

(- The Author(s). 2020 Open Access This article is licensed under a Creative Commons Attribution 4.0 International License, which permits use, sharing, adaptation, distribution and reproduction in any medium or format, as long as you give appropriate credit to the original author(s) and the source, provide a link to the Creative Commons licence, and indicate if changes were made. The images or other third party material in this article are included in the article's Creative Commons licence, unless indicated otherwise in a credit line to the material. If material is not included in the article's Creative Commons licence and your intended use is not permitted by statutory regulation or exceeds the permitted use, you will need to obtain permission directly from the copyright holder. To view a copy of this licence, visit http://creativecommons.org/licenses/by/4.0/. The Creative Commons Public Domain Dedication waiver (http://creativecommons.org/publicdomain/zero/1.0/) applies to the data made available in this article, unless otherwise stated in a credit line to the data. 


\section{Background}

'Subcutaneous partial rupture of the Achilles tendon' was first described in 1968 [1]. The author presented 24 cases and defined that entity as a 'tear involving a varying number of fibres in the free portion of the Achilles tendon, usually leaving most fibres intact' [1]. So far, only little research has been conducted. In a PubMed/ Medline internet search (20 March 2020), 79 articles were found for [Achilles tendon] and [partial] and [(tear) or (rupture)]. Twenty-one papers described imaging. There were 10 animal studies and two anatomic descriptions. Four papers presented overviews without original data. Thirteen studies focused on Achilles tendinopathy and nine on Achilles tendon ruptures. Eight publications were not relevant for the topic. Twelve clinical original case series and case studies presented 213 cases. From these, 83 lesions affected the midportion Achilles tendon, while 130 were located in the retrocalcaneal Achilles tendon area.

Clinical presentation of Achilles tendon partial tears is unspecific in most cases and is frequently not different from Achilles tendinopathy [1-3] or retrocalcaneal bursitis [4]. Suspicion of Achilles tendon partial tear is likely, when the patient experiences an acute onset, an audible pop, and a piercing pain during load [3, 5]. Dependent on the size and the age of the lesion, physical examination inconsistently presents Achilles tendon swelling or denting and calf muscle atrophy. Side differences in ankle dorsiflexion indicate Achilles tendon elongation in an advanced stage [6].

Diagnostic ultrasound imaging, colour Doppler, and/or MRI can underline the clinical suspicion [3, 7-9]. Ultrasound and power Doppler investigations demonstrated unspecific findings like localised swelling, reduced echogenicity, and neovascularisation related to the injured area $[3,7]$. More specific but inconsistent findings were discontinuity of tendon fibres and intratendineous anechogenic or low echogenic spots [3]. However, 'especially partial ruptures of the Achilles tendon' are not sufficiently detected by ultrasound [10]. MRI scans have the highest accuracy for Achilles tendon partial tears $[9,11]$.

Conservative treatment should initially be initiated and contain most modalities used also for Achilles tendinopathy $[12,13]$. However, caution against eccentric training is recommended, as it may increase the risk for total Achilles tendon rupture [2]. The reviewed literature presents only six (four midportion, two impingement) partial Achilles tendon tears with successful conservative therapy. When unresponsive to conservative therapy, operative procedures are recommended [1].

In the midportion area, Achilles tendon partial tears are operatively addressed by excision and side-to-side and/or end-to-end repair [1, 2, 4]. In the retrocalcaneal area, the bursa and the Haglund tuberosity are removed open or endoscopically. Some authors additionally repair this so-called impingement partial tear $[4,14,15]$ while others only excise the lesion $[16,17]$.

In a previous study, we compared results of operative treatment for Achilles tendinopathy and retrocalcaneal bursitis with or without Achilles tendon partial tears [4]. That study demonstrated no difference in outcome between the four respective groups, but the available numbers and the resulting power of that study were small.

The aim of this study was to compare the patientrelated outcome of operatively treated Achilles tendon partial tears when located in the midportion or in the retrocalcaneal area after a minimum of 42 months and at 3, 6, and 12 months. Besides, the VISA-A-G (Victorian Institute of Sports Assessment-Achilles tendon, German version) outcomes within the groups were compared from preoperative to 3, 6, 12, and more than 42 months postoperative.

\section{Material and methods \\ Ethics}

The Landesärztekammer Hessen Ethics Committee (FF 162/2016) approved this study.

Informed consent was obtained from all patients, and the rights of the patients were protected. The registration trial number is DRKS00014266 on DRKS. 'Retrospectively registered'. Date of registration: 06 April 2018.

\section{Patients and grouping}

The patients of this study were operated between November 2009 and end of 2015. We searched our electronic databases for patients operated for Achilles tendon partial tear. Dependent on the anatomic level of the Achilles tendon partial tear and from the respective operative procedure, we enrolled patients either to a 'midportion group' or to a 'retrocalcaneal group' (Fig. 1 [18]). To be included, patients preoperatively had to be unresponsive to two or more of the following conservative treatment modalities: load modification, rest, acupuncture, orthotics, bandage, NSAID, eccentric exercises, physiotherapy, ice, ESWT, injections, and radiation. The analyses comprised only datasets of patients, who responded to the final follow-up questionnaire. Two patients included, both from the 'retrocalcaneal group', underwent bilateral operations within 3 weeks and 2 months. The respective final follow-up VISA-A-G scores for these four lower extremities were 100, 100, 100, and 88. To avoid 'double dipping' effects [19] only the scores obtained from the sides operated on first were included (100 and 88).

Further exclusion criteria were no partial tear described in the operative report $(n=1)$, previous Achilles tendon surgery $(n=8)$, additional procedures performed during surgery $(n=5)$, unwilling to participate $(n=1)$, 


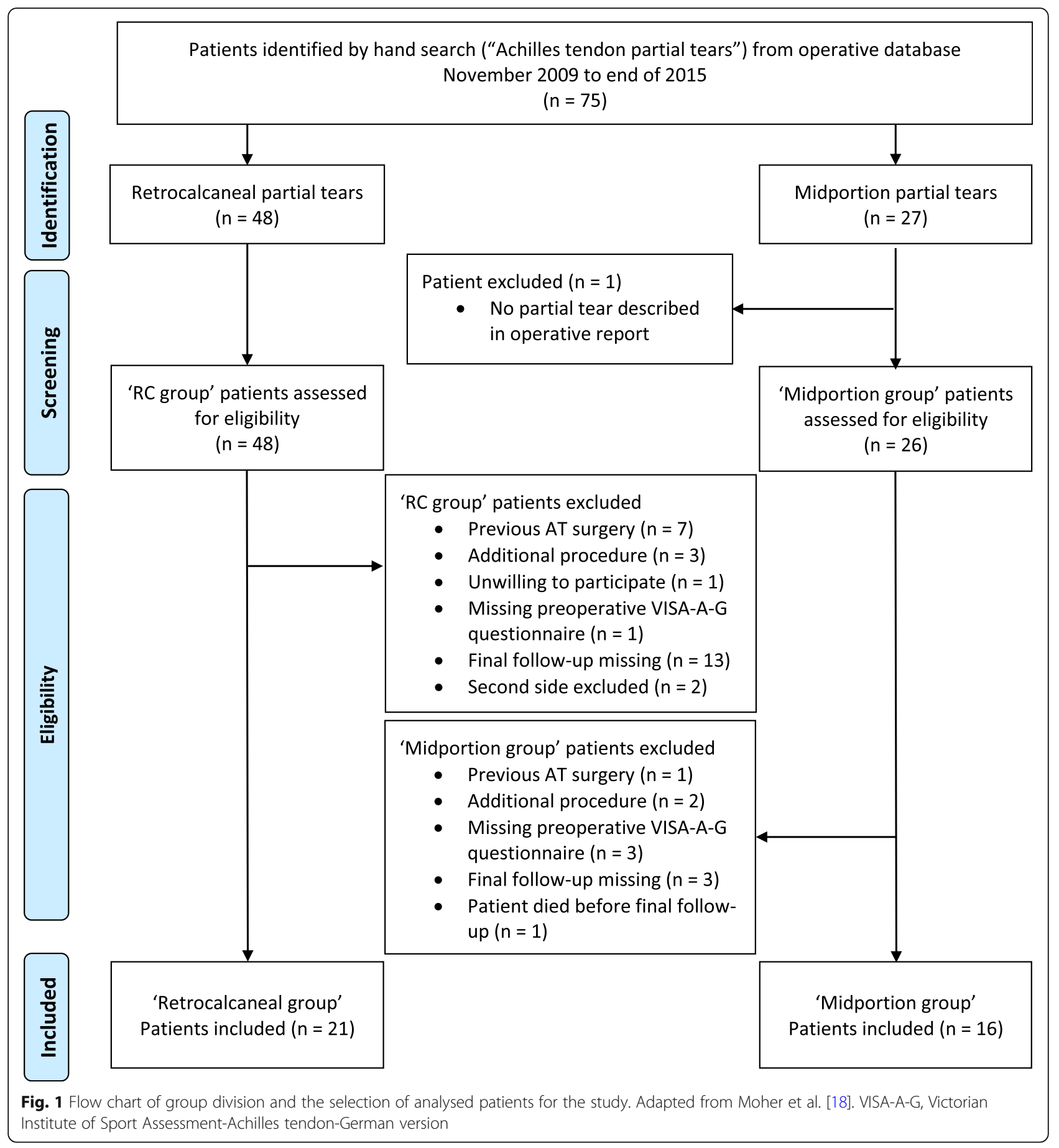

missing preoperative VISA-A-G questionnaire $(n=4)$, final follow-up missing $(n=16)$, and second side excluded $(n=2)$. One patient died between the 12 months and the final follow-up for reasons not related to the Achilles tendon surgery. Three midportion and three retrocalcaneal Achilles tendon partial tear patients (operated between 2009 and 2011) were already enrolled in a previous evaluation [4].

\section{Diagnostics}

History was nonspecific in most cases and was not different from Achilles tendinopathy or chronic retrocalcaneal bursitis. Patients generally complained about sport/ running-induced pain in the involved Achilles tendon. This pain increased over time and increasingly limited the patients' activity. In typical cases, an acute event exacerbated the symptoms (Table 1). 


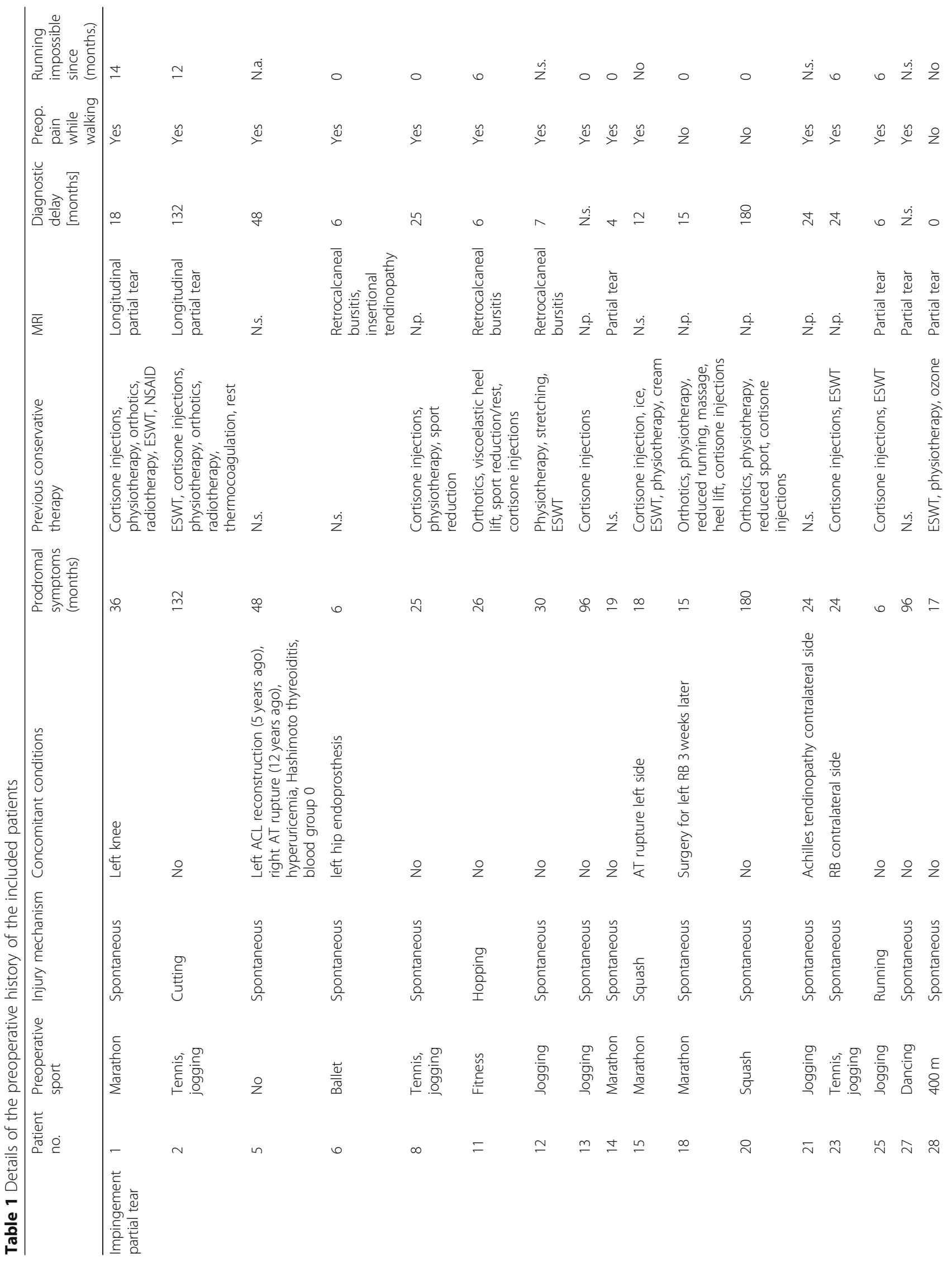




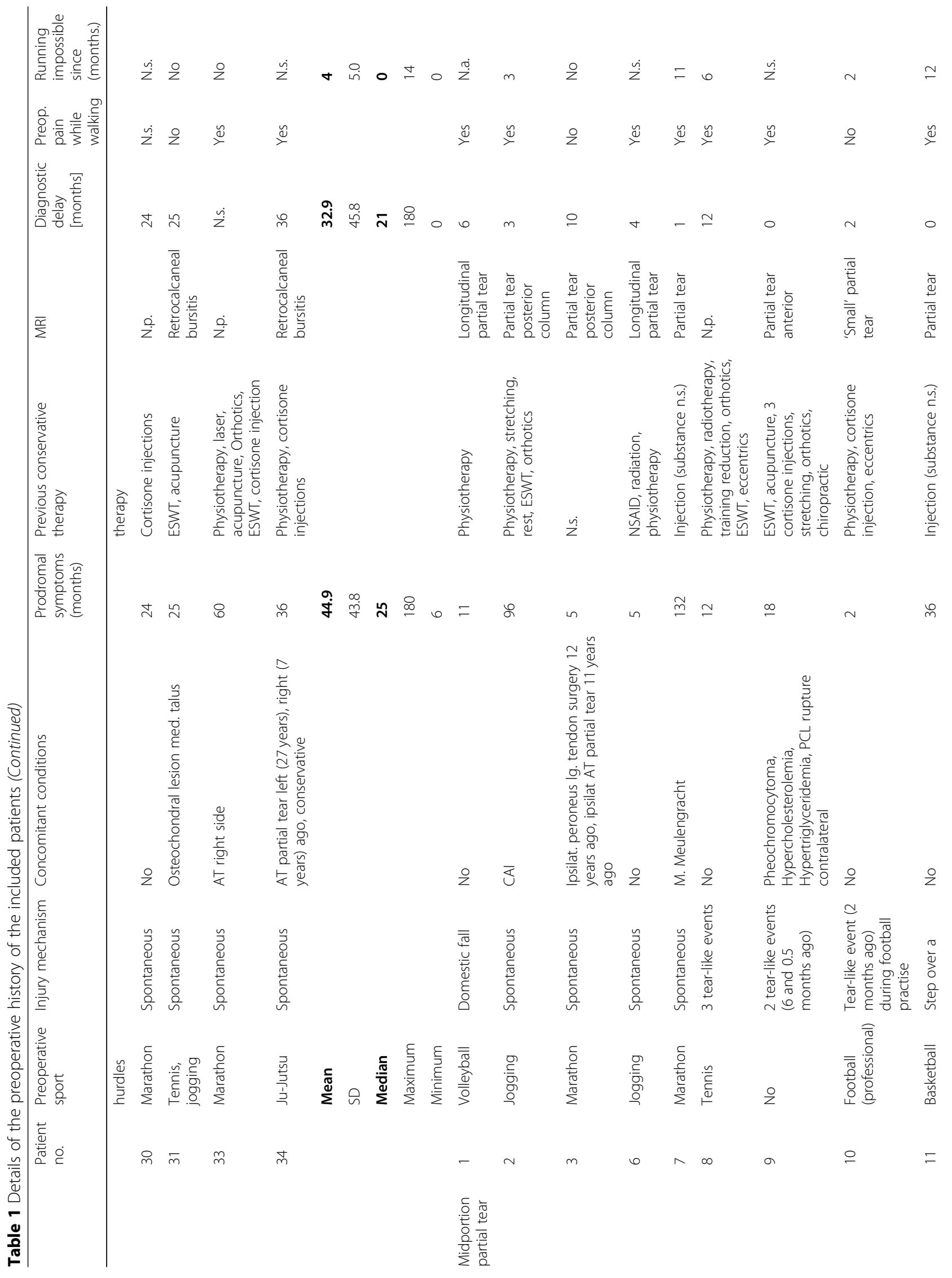




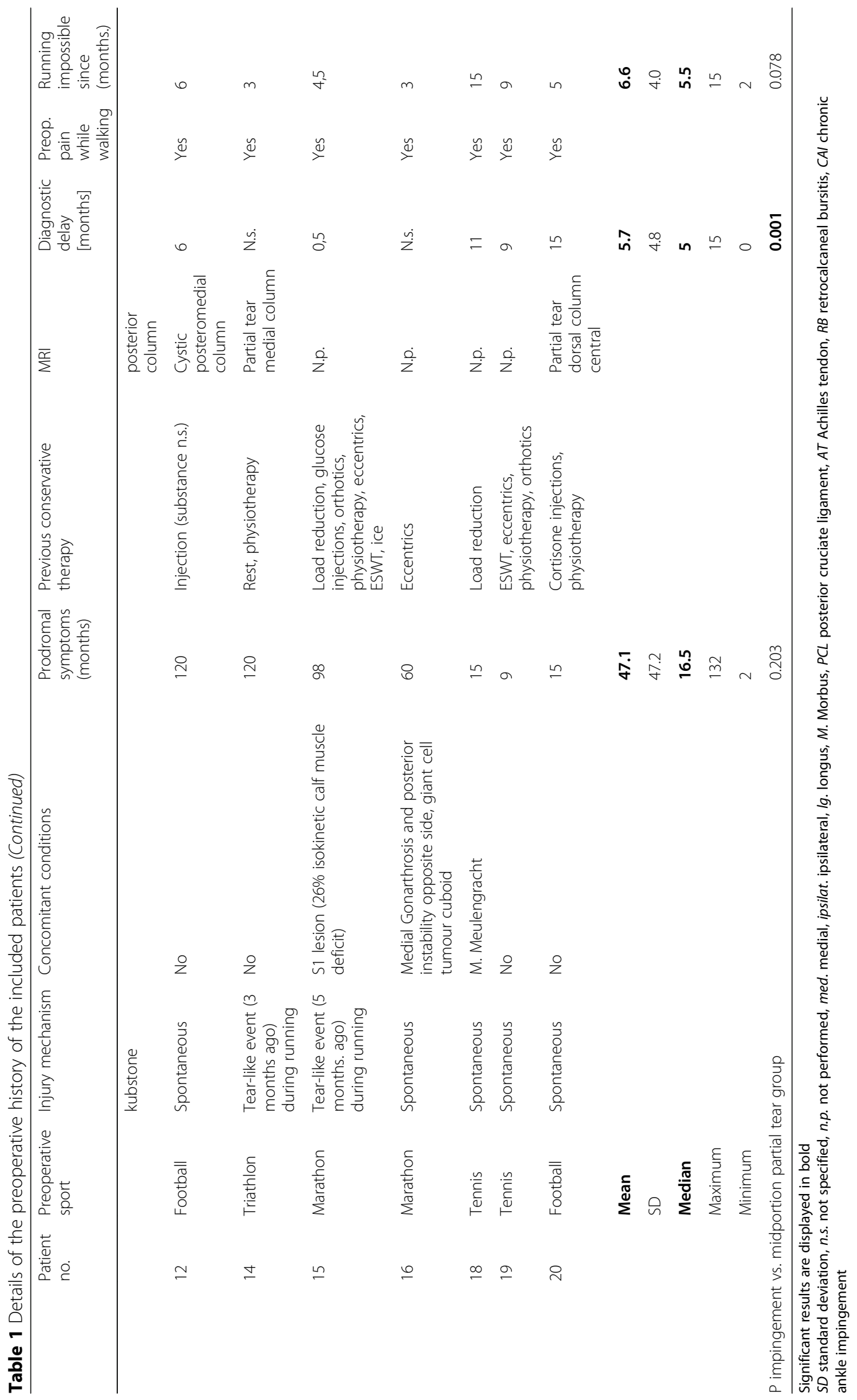


Physical examination allocated the patient's symptoms to the injured Achilles tendon region. The most important finding was the localised pain on palpation related either to the Achilles tendon midportion or to the retrocalcaneal area. Midportion lesions additionally demonstrated a spindle-shaped swelling at the Achilles tendon $2-7 \mathrm{~cm}$ above its calcaneal insertion. Retrocalcaneal lesions presented with swelling related to the Haglund/retrocalcaneal region.

All patients underwent ultrasound and power Doppler investigations, and MRI scans were available for $21 \mathrm{pa}-$ tients (Table 1).

\section{Operative procedures}

A single orthopaedic surgeon performed all procedures. The operative techniques for the two different lesions have already been described in detail [4]. Midportion Achilles tendon partial tears were accessed by transverse or longitudinal skin incisions. The paratenon was resected, and the anterior Achilles tendon was released. Following longitudinal splitting of the Achilles tendon, the lesion was identified and excised. Repair comprised transverse anterior O-shaped side-to-side and a posterior running suture (2-0 and 3-0 Vicryl). In five cases, a plantaris tendon transplant reinforced the reconstruction. For retrocalcaneal Achilles tendon partial tears, an oblique to transverse or a longitudinal skin incision at the lateral Achilles tendon border was made over the lateral aspect of the retrocalcaneal bursa. At the lateral Achilles tendon border, the retrocalcaneal bursa was accessed by a longitudinal incision. The subcutaneous bursa, the retrocalcaneal bursa, and Haglund's tuberosity were removed from lateral to medial. By elevating the lateral Achilles tendon border, the anterior Achilles tendon partial tear was identified and debrided, and the lesion was repaired with one to five $\mathrm{Z}$ - or O-shaped transverse 2-0 Vicryl sutures [15].

\section{Postoperative care}

Postoperative treatment and rehabilitation regimen was not different for the two groups. Postoperatively, a Scotchcast splint was applied for 3-4 days and remained for 4 weeks postoperatively as a night splint. A stable shoe with a 1.5-2-cm heel lift was initiated 3-4 days postoperatively during the day, and load was gradually increased during the following 1-2 days. About 6 weeks postoperatively, the heel lift was reduced to $1 \mathrm{~cm}$. The stable shoe was discontinued 6 weeks postoperatively but the $1-\mathrm{cm}$ heel lift remained in the patient's normal shoe for six more weeks. After the 12th postoperative week, the patient, if free of pain, was allowed to gradually commence running activities. If symptom free, full load in practice and during competitions were allowed 6 months postoperatively.

\section{Follow-up}

There is no validated research tool available for Achilles tendon partial tears. Therefore, outcome was prospectively evaluated using the VISA-A-G questionnaire preoperatively (baseline) and 3, 6, and 12 months and after a minimum of 3.5 years postoperatively. The VISAA questionnaire is the only valid, reliable, and diseasespecific patient-administered questionnaire for research in Achilles tendinopathy [20-22]. It measures the severity of pain and function, related to activities of daily living (six items) and during sport (two items). A score of 0 means a maximal impairment and 100 reflects an asymptomatic person. In principle, the VISA-A questionnaire is designed and validated only for Achilles tendinopathy in the midportion and/or retrocalcaneal area [20-22]. However, the VISA-A score correlates with the ATRS (Achilles tendon Total Rupture Score) [23]. Both tools are not specifically validated for Achilles tendon partial tears, and the ATRS is not available in German language. We therefore decided to use the VISA-A-G questionnaire as the best suitable tool for this study. Two patients (one in each group) were not involved in any sport. Corresponding to the proposed procedure [24], their results were calculated from the percentage result of questions 1 to 6 only.

Two patients in the retrocalcaneal group underwent bilateral operations ( 3 weeks and 2 months interval). For these patients, only the results for the side of the initial intervention were further analysed.

A VISA-A-G score of 90 and more was regarded as excellent, 70-89 as good, and below 70 as unsuccessful [25]. From this classification, success rate is defined as the summarised excellent and good results $[4,26]$.

The clinical records were retrospectively screened for the clinical status, including ultrasound and MRI (if applicable). The clinical appointments were conducted according to the individual rehabilitation process of the patients. A specific clinical and imaging follow-up was not scheduled. Power Doppler ultrasound results were graded according to previous research [27].

\section{Statistical analysis}

Inferential statistical analysis was carried out using SPSS 26.0 (IBM Inc., USA). Repeated measures one way ANOVA was conducted for the VISA-A-G score to identify between group differences, time effects, and group $\mathrm{x}$ time interaction effects. For $9 \%$ missing values, the last observation carried forward technique has been applied. Further evaluated parameters (anthropometry, pre- and postoperative clinical data) were compared descriptively between groups or, if applicable, analysis using the unpaired $T$ test for normally distributed parameters or the Mann-Whitney $U$ test for non-normally distributed values (extent of resection, initiation of 
running activities postoperatively). The significance level was set at $p=0.05$. Results are reported as means with corresponding standard deviations (SD) and ranges.

\section{Results}

\section{VISA-A-G outcome}

There was homogeneity of covariances, as assessed by Box's test $(p=0.053)$. Mauchly's test of sphericity indicated that the assumption of sphericity had not been violated, $X^{2}(9)=12.042, p=0.211$. A repeated measures ANOVA determined that mean performance levels showed a statistically significant difference between measurements, $\mathrm{F}(4,140)=73.40, p<0.001$, partial $\eta^{2}=0.68$ (Tables 2, 3, and 4). There was no significant main effect for group, $\mathrm{F}(1,35)=1.979, p<0.168$, partial $\eta^{2}=0.054$. There was no statistically significant interaction between time and group, $\mathrm{F}(4,140)=0.361, p=0.836$ (Table 2).

\section{Success rates}

At final follow-up, excellent results/full recoveries in the retrocalcaneal partial tear and the midportion partial tear group were found in 14/21 (67\%) and 15/16 (94\%) cases, respectively. Good results were found in $5 / 21$ (24\%) and 1/16 (6\%) patients in the retrocalcaneal partial tear and the midportion partial tear group, respectively. Unsuccessful outcome at final follow-up was found in 2/ $21(9.5 \%)$ patient in the retrocalcaneal partial tear group. The success rate is $91 \%$ for retrocalcaneal partial tear and $100 \%$ for the midportion partial tear group.

\section{Anthropometric data}

There were 11 males and 10 females within the retrocalcaneal partial tear group, while the midportion partial tear group comprised 15 males and 1 female. Patients' age at surgery in the retrocalcaneal partial tear and in the midportion partial tear group was $51 \pm 9.2$ (range, 20-65) and $50 \pm 9.3$ (range, 23-66) years $(p=0.724)$, respectively. Patients' height in the retrocalcaneal partial tear and in the midportion partial tear group was $176 \pm$ 9.4 (range, 158-192) and $183 \pm 6.1$ (range, 174-193) $\mathrm{cm}$ $(p=0.013)$, respectively. Patients' weight in the retrocalcaneal partial tear and in the midportion partial tear group was $74 \pm 15.5$ (range, 47-115) and $83 \pm 11.6$ (range, 65-110) $\mathrm{kg}(p=0.046)$, respectively. BMI for patients in the retrocalcaneal partial tear and in the midportion partial tear group was $24 \pm 3.8$ (range, 19-36) and $25 \pm 3.1$ (range, 21-32) $\mathrm{kg}(\mathrm{p}=0.307)$, respectively.

\section{Preoperative history}

In the retrocalcaneal and in the midportion partial tear group, the left/right Achilles tendon was affected in 10/ 11 and 8/8 patients, respectively. Two patients of the retrocalcaneal partial tear group had bilateral involvement with surgery performed during the study period but only the side operated on first was considered for further evaluation, due to our exclusion criteria.

Only one patient in either group was not involved in regular sports. Running activities (21/37), tennis (7/37 patients), and football (3/37 patients) were the predominant preoperative sports (Table 1). Achilles tendon symptoms developed insidiously in $17 / 21$ (81\%) and 9/ $16(56 \%)$ in the retrocalcaneal partial tear and in the midportion partial tear group, respectively (Table 1). No preceding injuries involving the injured Achilles tendon or systemic medical conditions were specified in 11/21 (52\%) patients with retrocalcaneal partial tears and 9/16 (56\%) with midportion partial tears. Patients in the retrocalcaneal partial tear and in the midportion partial tear group described prodromal symptoms for $44.9 \pm$ 43.8 (range, 6-180) and $47.1 \pm 47.2$ (range, 2-132) months $(p=0.203)$, respectively. Patients preoperatively underwent different forms of conservative treatment (Table 1). Preoperative MRI correctly detected 6/11 retrocalcaneal impingement partial tears and diagnosed 5/ 11 patients in the retrocalcaneal partial tear group as suffering from retrocalcaneal bursitis. In the midportion partial tear group, MRI correctly identified 10/11 midportion Achilles tendon partial tears and diagnosed 1/11 'cystic posteromedial column'. The period from onset of symptoms to diagnosis was $32.9 \pm 45.8$ (range, $0-180$ ) and $5.7 \pm 4.8$ (range, $0-15)$ months $(p=0.001)$ in the retrocalcaneal partial tear and in the midportion partial tear group, respectively (Table 1 ). Preoperatively, 16/20 $(80 \%)$ and $14 / 16(88 \%)$ in the retrocalcaneal partial tear and in the midportion partial tear group, respectively, complained about Achilles tendon pain while walking (Table 1). Running activities were preoperatively impossible due to the symptoms since $4.0 \pm 5.0$ (range, $0-14$ ) and $6.6 \pm 4.0$ (range, $2-15)$ months $(p=0.078)$ in the retrocalcaneal partial tear and in the midportion partial tear group, respectively (Table 1 ).

\section{Intraoperative findings}

All retrocalcaneal partial tears were addressed by retrocalcaneal bursa and Haglund resection, debridement, and repair of the partial tears. All midportion partial tears were debrided and repaired side to side. In 5/16 (31\%) of those patients, a plantaris tendon augmentation was added. About $14.3 \pm 7.8$ (range, 5-40) and $24.7 \pm$ 18.7 (range, 5-60)\% of the local tendons' cross-section area were excised in the retrocalcaneal partial tear and midportion partial tear group, respectively $(p=0.125$; Table 2).

\section{Complications}

No major complications occurred in both groups. In the retrocalcaneal partial tear and in the midportion partial tear group, 5/16 (31\%) and 4/19 (21\%) of the patients, 


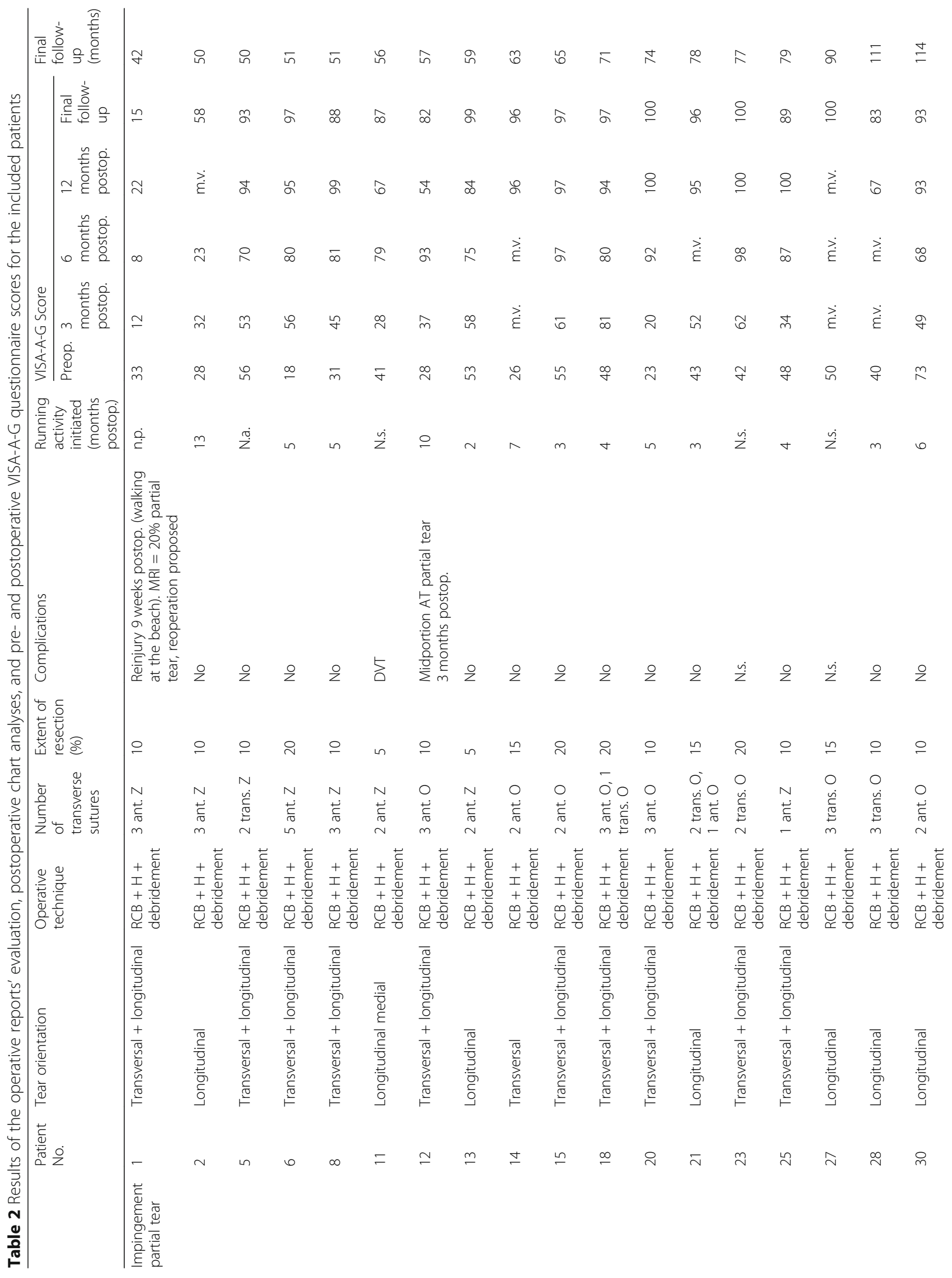




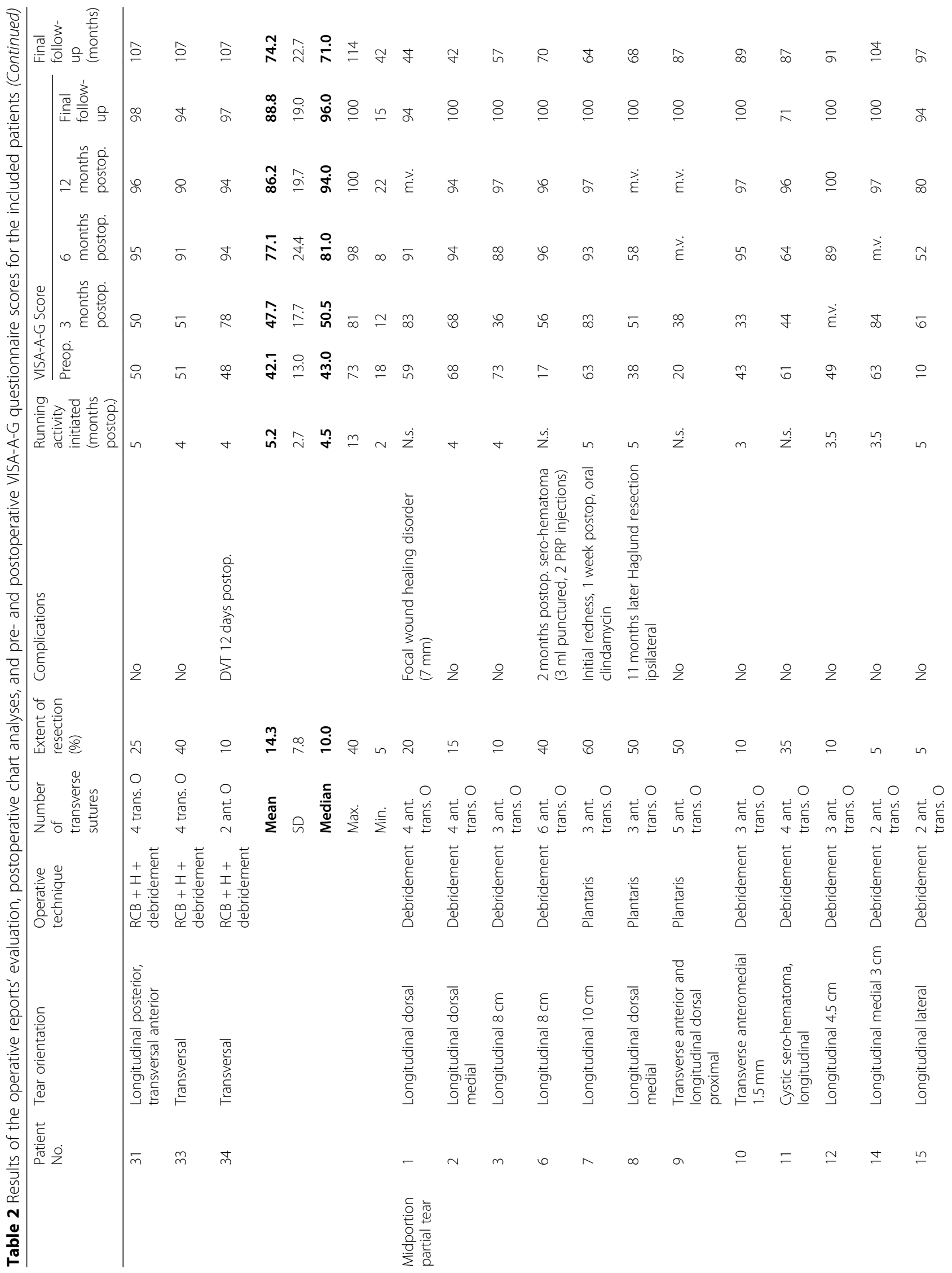




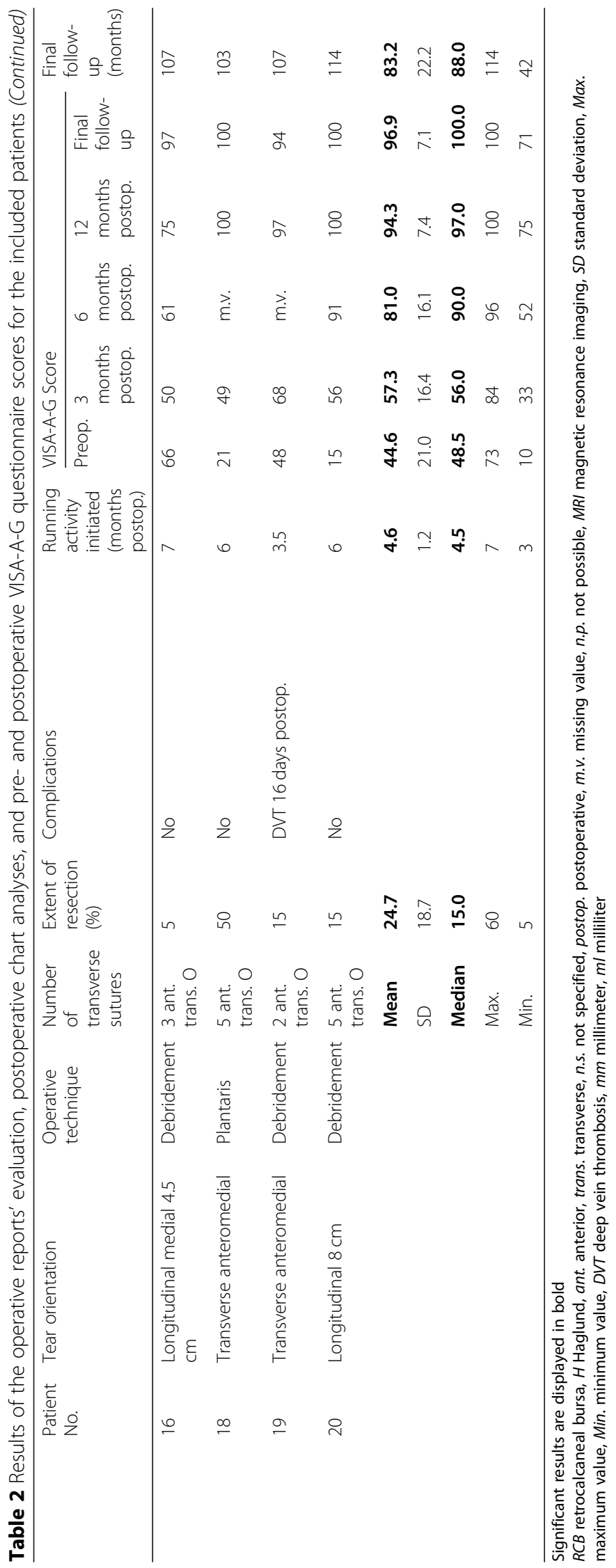


Table 3 Longitudinal ANOVA comparison of the VISA-A-G results for the retrocalcaneal partial tear group

\begin{tabular}{|c|c|c|c|c|}
\hline & 3 months postop. & 6 months postop. & 12 months postop. & Final follow-up \\
\hline Preoperative & 1.000 & 0.001 & $<0.001$ & $<0.001$ \\
\hline 3 months postop. & & 0.001 & $<0.001$ & $<0.001$ \\
\hline 6 months postop. & & & 0.281 & 0.004 \\
\hline 12 months postop. & & & & 0.368 \\
\hline
\end{tabular}

Significant results are displayed in bold

Postop. postoperative

respectively, suffered from postoperative complications, including two and one deep vein thromboses, respectively. One non-compliant patient resumed jogging already 10 weeks after retrocalcaneal partial tear surgery without permission and had a reinjury, which was conservatively treated. One additional patient complained about continuing postoperative pain following a barefoot walk on the beach at 9 weeks postoperatively. One year postoperatively, a reoperation was proposed, but the patient refused. He scored the lowest VISA-A-G value (15 points) at the final follow-up (Table 2).

Postoperative running activities were resumed $5.2 \pm$ 2.7 (range, 2-13) and $4.6 \pm 1.2$ (range, 3-7) months in the retrocalcaneal partial tear and in the midportion partial tear group, respectively ( $p=0.492$; Table 2$)$.

\section{Presentation at last clinical examination}

The final clinical investigations were held 10 (range 0-69) and 6.5 (1-23) months postoperatively for the retrocalcaneal partial tear and the midportion partial tear group, respectively $(p=0.624)$. At final clinical investigation, tenderness in the operated Achilles tendon area was still found in 4/18 (22.2\%) and 5/16 (31.3\%) in the retrocalcaneal partial tear and the midportion partial tear patients, respectively. Due to persisting pain, postoperative MRI investigations were performed in two and one patients of the retrocalcaneal partial tear and the midportion partial tear group, respectively. Ultrasound revealed power Doppler grade $0-$ II in 14/18 (77.8\%) and 13/16 (81.3\%) of the patients in the retrocalcaneal partial tear and the midportion partial tear group, respectively, while grade III or IV were found in 4/18 (22.2\%) and 3/16 (18.8\%). In the midportion partial tear group, Achilles tendon sagittal diameter was 12 \pm 3.4 (range, $7-18 \mathrm{~mm}$ ). Respective values were not recorded for the retrocalcaneal partial tear group.

\section{Discussion}

This study demonstrates that open surgery for Achilles tendon partial tears when recalcitrant to conservative treatment leads to excellent results in more than two thirds of our patients, irrespective of the anatomic location of the injury. Postoperative recovery is slow in both entities. This is underlined by the fact that no statistically relevant improvement could be detected between the preoperative and the 3 month postoperative result. From then, evidence for improvement is provided until 1 year postoperatively, while later, a further improvement of the status could not be verified for either group (Tables 3 and 4).

A minimum important clinical difference of 6.5 VISAA points was formally established for 'insertional Achilles tendinopathy' [28]. The mean between group differences were more than 6.5 points at 3 and 12 months and at final follow-up, indicating a tendency towards better outcomes in patients suffering from midportion Achilles tendon partial tears.

The between group differences in height and weight of the patients are a result of the nearly equal (52\% male) sex distribution in the retrocalcaneal partial tear group while in the midportion partial tear group only one out of 16 patients (6\%) was female. BMI was statistically not different between groups.

Interestingly, there was no bilateral involvement in the midportion partial tear group, but 3/21 (14\%) of the retrocalcaneal partial tear group had bilateral involvement during the study period. Nearly all patients were active in sport, and in most instances, the patient's history revealed a specific initiating event.

Associated pathologies may play a predisposing role for Achilles tendon partial tears. In 90.9\%, foot pain was associated with joint pain at other sites [29].

Table 4 Longitudinal ANOVA comparison of the VISA-A-G results for the midportion partial tear group

\begin{tabular}{lllll}
\hline & 3 months postop. & 6 months postop. & 12 months postop. & Final follow-up \\
\hline Preoperative & 0.589 & $\mathbf{0 . 0 0 1}$ & $<\mathbf{0 . 0 0 1}$ & $<\mathbf{0 . 0 0 1}$ \\
3 months postop. & & $\mathbf{0 . 0 2 9}$ & $\mathbf{0 . 0 4 2}$ & $\mathbf{0 . 0 0 1}$ \\
6 months postop. & & $\mathbf{0 . 0 0 5}$ & $\mathbf{0 . 0 0 5}$ \\
12 months postop. & & & 1.000 \\
\hline
\end{tabular}


Correspondingly, in both groups of this study, additional preceding injuries to different parts of the body and systemic medical conditions were frequent. Further research should therefore address the pathogenetic relevance of these comorbidities and its possible influence to the VISA-A scores.

Diagnosis of the described conditions is frequently delayed, ranging from 1 to 180 months, but the midportion Achilles tendon partial tears are diagnosed earlier (median $=5$ vs. 21 months, $p=0.001$ ). The analysed data cannot explain this difference. The lower chronic status of the injured midportion Achilles tendons, however, may be responsible for the between group difference 12 months postoperatively and at final follow-up. In contrast, a previous study found no statistically relevant difference between the 12-month results of partial tears in the midportion and retrocalcaneal area [30].

The role of local cortisone injections during the preceding conservative treatment of Achilles tendinopathy and retrocalcaneal bursitis is a matter of debate. Systematic research does not support injection therapy in general [31]. 'Long-term harms to tendon tissue and cells associated with glucocorticoid injections' are assumed [32], also following injections into the retrocalcaneal bursa [33]. In our retrocalcaneal and midportion partial tear group 13/21 (62\%) and 3/16 (19\%) of the respective patients had previous cortisone injections.

Literature evaluating partial Achilles tendon tears is rare. It is to assume, that the initial lesion for retrocalcaneal partial tear is impingement resulting from retrocalcaneal bursitis [14, 15, 30, 34].

The strength of this study is that a single orthopaedic surgeon performed all procedures in a standardised manner. Rigorous inclusion and exclusion criteria produced well-defined groups for comparison with the so far longest follow-up. Another strength is the longitudinal design to demonstrate that interval improvement of the injured Achilles tendons at any level is slow and requires about 1 year.

There are inherent limitations to this study. There are low numbers in the groups. Therefore, the tendency towards superiority of the midportion Achilles tendon partial tear group is not robust enough, and larger groups are required to further underline these results. In principle, a selection bias could arise when patients with good results would be more willing to answer the VISAA-G questionnaire. Consequently, excluding patients with a missing final follow-up could lead to positively overestimating the results. However, there was no statistical difference between the 12 months and final follow-up results between all patients who completed the 12-month questionnaire and those recruited for further calculations (all $p>0.195$ ). Finally, relying on a patient related and therefore subjective outcome measure could be criticised. However, the VISA-A is 'a valid and reliable index of the clinical severity of Achilles tendinopathy' [22]. It is proposed for 'clinical measurement studies' [35]. Since its development, it was crossculturally translated and adapted to all major languages and is globally accepted [25, 36]. This is important to make results of different researchers internationally comparable. Additionally, it 'seems suitable for both clinical rating and quantitative research' [37]. Endoscopic interventions are becoming more and more popular for retrocalcaneal bursitis/Haglund's syndrome, and good results are reported also when impingement Achilles tendon lesions were addressed merely by retrocalcaneal bursa and Haglund resection [16, 17]. Direct experimental comparison of endoscopic and open retrocalcaneal bursitis and Haglund resection did not reveal an advantage of one technique over the other [38]. Endoscopic repair techniques are not described for midportion and retrocalcaneal Achilles tendon partial tears so far. Therefore, further clinical research should compare open and endoscopic procedures for partial Achilles tendon tears in the midportion and retrocalcaneal area using standardised procedures in a controlled and randomised design.

Rare case reports document successful conservative treatment of Achilles tendon partial tears in the retrocalcaneal and midportion area [6, 12, 39-41]. The current study included only patients who were unresponsive to conservative treatment. Experimental work in a rat model demonstrated that injury severity had a drastic influence on biomechanical characteristics of the Achilles tendons [42]. It can be speculated, that minor partial tears may be more responsive to conservative treatment modalities. Probably, patients with low functional demands respond better to conservative treatment.

Postoperative care may have an influence on outcome. Patients of both groups in this investigation underwent early functional treatment and wore heel lifts in rehabilitation boots for several weeks. In rat experiments, immobilisation reduced function and fatigue resistance of Achilles tendons with partial tears post-injury [42]. Further clinical studies can demonstrate if this association can be transferred to Achilles tendon partial tears in humans.

MRI and diagnostic ultrasound is 'used to identify or to confirm' Achilles tendon partial tears and for distinguishing it from complete ruptures and tendinosis [9]. It is recommended for postoperative care [9]. MRI and ultrasonography can confirm the diagnosis but do not consistently detect partial tears of the Achilles tendons. Specifically, the sensitivity of MRI for diagnosing impingement partial tears is not sufficient in this study (Table 1). This finding underlines previous research, demonstrating that 'Ultrasound and MRI show only 
moderate correlation with clinical assessment of chronic Achilles tendinopathy' [43]. Neither for MRI nor for diagnostic ultrasound a grading system exists to evaluate postoperative Achilles tendons. In a recent study 'intratendinous' tears were introduced in the differential diagnosis of Achilles tendon disorders. That pathology was ultrasonographically differentiated from partial tears by fibre discontinuity 'entirely within' the tendon [3].

\section{Conclusion}

Achilles tendon partial tears can occur in the midportion area and at the level of the retrocalcaneal bursa. In recalcitrant cases, operative treatment is successful in most cases. VISA-A-G questionnaire demonstrated increasing functionality and decreasing symptoms during the first postoperative year, and results do not deteriorate in the long-term.

\section{Abbreviations \\ VISA-A-G: Victorian Institute of Sports Assessment-Achilles tendon-German version}

\section{Acknowledgements}

The author is grateful to Mrs. Erica Rademacher and Mrs. Denise Rademacher for their valuable help in professional language editing of the paper as native English speakers.

Sincere thanks are given to PD Dr. Dominic Gehring (Department of Sport and Sport Science, Albert-Ludwigs-Universität Freiburg, Germany) for his valuable statistical advice during the revision of the manuscript and to Stephanie Wagner (European SportsCare Network, Wiesbaden) for performing these analyses.

I would like to thank Mrs. Maria Scherer and Mrs. Claudia Werner for sending and administering the VISA-A-G questionnaires.

The article-processing charge was funded by the German Research Foundation (DFG) and the University of Freiburg in the funding programme Open Access Publishing.

\section{Author's contributions}

The author conceived and designed the study, performed data acquisition, interpreted the data, and drafted the manuscript. The author read and approved the final manuscript.

\section{Funding}

This paper received no specific grant from any funding agency in the public, commercial, or non-profit sectors.

\section{Availability of data and materials}

The datasets used and analysed during the current study are available from the author on reasonable request.

\section{Ethics approval and consent to participate}

The Landesärztekammer Hessen Ethics Committee approved this study (FF 162/2016)

\section{Consent for publication}

The patients consented (by signature) for publication of their material in this study.

\section{Competing interests}

The author declares that he is a paid speaker for Orthotech GmbH, Gauting Germany.
Received: 15 April 2020 Accepted: 31 July 2020

Published online: 10 September 2020

\section{References}

1. Ljungqvist R. Subcutaneous partial rupture of the Achilles tendon. Acta Orthop Scand 1967; Suppl.

2. Alfredson $\mathrm{H}$, Spang C. Clinical presentation and surgical management of chronic Achilles tendon disorders - a retrospective observation on a set of consecutive patients being operated by the same orthopedic surgeon. Foot Ankle Surg. 2017.

3. Chan O, Morton S, Pritchard M, et al. Intratendinous tears of the Achilles tendon - a new pathology? Analysis of a large 4-year cohort. Muscles Ligaments Tendons J. 2017;7:53-61.

4. Lohrer $\mathrm{H}$, Nauck T. Results of operative treatment for recalcitrant retrocalcaneal bursitis and midportion Achilles tendinopathy in athletes. Arch Orthop Trauma Surg. 2014;134:1073-81.

5. Skeoch DU. Spontaneous partial subcutaneous ruptures of the tendo achillis. Review of the literature and evaluation of 16 involved tendons. Am J Sports Med. 1981;9:20-2.

6. So V, Pollard H. Management of Achilles tendon disorders. A case review. Australas Chiropr Osteopathy. 1997;6:58-62.

7. Alfredson H, Masci L, Ohberg L. Partial mid-portion Achilles tendon ruptures: new sonographic findings helpful for diagnosis. Br J Sports Med. 2011;45: 429-32.

8. Alghamdi N, Killian M, Aitha B, et al. Quantifying the dimensions of Achilles tendon insertional area using ultrasound imaging a validity and reliability study. Muscle Ligaments and Tendons Journal. 2019;09:544-51.

9. Oliva F, Rugiero C, Giai Via A, et al. I.S.Mu.L.T. Achilles tendon ruptures guidelines. Muscles Ligaments Tendons J. 2018;08:310-63.

10. Kayser R, Mahlfeld K, Heyde CE. Partial rupture of the proximal Achilles tendon: a differential diagnostic problem in ultrasound imaging. $\mathrm{Br} J$ Sports Med. 2005;39:838-42.

11. Weinstabl R, Stiskal P, Neuhold A, Hertz H. MR and ultrasound study of Achilles tendon injury. Unfallchirurgie. 1992;18:213-7.

12. Hsu YC, Wu WT, Chang KV, et al. Healing of Achilles tendon partial tear following focused shockwave: a case report and literature review. J Pain Res. 2017:10:1201-6.

13. Kvist M. Achilles tendon injuries in athletes. Sports Med. 1994;18:173-201.

14. Lohrer $\mathrm{H}$, Arentz S. Impingement lesion of the distal anterior Achilles tendon in sub-Achilles bursitis and Haglund-pseudoexostosis-a therapeutic challenge. Sportverletz Sportschaden. 2003;17:181-8.

15. Lohrer $\mathrm{H}$. Minimally invasive repair of an impingement induced partial tear of the anterior Achilles tendon in a top level athlete. Z Orthop Unfall. 2010; 148:80-2.

16. Jerosch J, Sokkar S, Ducker M, Donner A. Endoscopic calcaneoplasty (ECP) in Haglund's syndrome. Indication, surgical technique, surgical findings and results. Z Orthop Unfall. 2012;150:250-6.

17. Pasa L, Kuzma J, Herufek R, et al. Arthroscopic treatment of chronic retrocalcaneal bursitis - endoscopic calcaneoplasty. Acta Chir Orthop Traumatol Cechoslov. 2018:85:209-15.

18. Moher D, Liberati A, Tetzlaff J, Altman DG. Preferred reporting items for systematic reviews and meta-analyses: the PRISMA statement. J Clin Epidemiol. 2009;62:1006-12.

19. Derr J. Valid paired data designs: make full use of the data without "doubledipping". J Orthop Sports Phys Ther. 2006;36:42-4.

20. Lohrer H, Nauck T. Cross-cultural adaptation and validation of the VISA-A questionnaire for German-speaking Achilles tendinopathy patients. BMC Musculoskelet Disord. 2009;10:134.

21. Lohrer H, Nauck T. Validation of the VISA-A-G questionnaire for Germanspeaking patients suffering from Haglund's disease. Sportverletz Sportschaden. 2010:24:98-106.

22. Robinson JM, Cook JL, Purdam C, et al. The VISA-A questionnaire: a valid and reliable index of the clinical severity of Achilles tendinopathy. $\mathrm{Br} \mathrm{J}$ Sports Med. 2001;35:335-41.

23. Nilsson-Helander $K$, Thomee $R$, Silbernagel KG, et al. The Achilles tendon Total Rupture Score (ATRS): development and validation. Am J Sports Med. 2007;35:421-6.

24. Sierevelt I. van $\mathrm{SM}$, Tol $\mathrm{H}$, et al. Dutch version of the Victorian Institute of Sports Assessment-Achilles questionnaire for Achilles tendinopathy: reliability, validity and applicability to non-athletes. World J Orthop. 2018;9:1-6. 
25. Iversen JV, Bartels EM, Langberg $H$. The Victorian Institute of Sports Assessment - Achilles questionnaire (VISA-A) - a reliable tool for measuring Achilles tendinopathy. Int J Sports Phys Ther. 2012;7:76-84.

26. Tallon C, Coleman BD, Khan KM, Maffulli N. Outcome of surgery for chronic Achilles tendinopathy. A critical review. Am J Sports Med. 2001;29:315-20.

27. Ohberg L, Alfredson H. Ultrasound guided sclerosis of neovessels in painful chronic Achilles tendinosis: pilot study of a new treatment. Br I Sports Med. 2002;36:173-5.

28. McCormack J, Underwood F, Slaven E, Cappaert T. The minimum clinically important difference on the VISA-A and LEFS for patients with insertional Achilles tendinopathy. Int J Sports Phys Ther. 2015;10:639-44.

29. Keenan AM, Drake C, Conaghan PG, Tennant A. The prevalence and impact of self-reported foot and ankle pain in the over 55 age group: a secondary data analysis from a large community sample. J Foot Ankle Res. 2019;12:53.

30. Lohrer $\mathrm{H}$, Nauck T. Retrocalcaneal bursitis but not Achilles tendinopathy is characterized by increased pressure in the retrocalcaneal bursa. Clin Biomech (Bristol, Avon). 2014;29:283-8.

31. Kearney RS, Parsons N, Metcalfe D, Costa ML. Injection therapies for Achilles tendinopathy. Cochrane Database Syst Rev. 2015:CD010960.

32. Dean BJ, Lostis E, Oakley T, et al. The risks and benefits of glucocorticoid treatment for tendinopathy: a systematic review of the effects of local glucocorticoid on tendon. Semin Arthritis Rheum. 2014;43:570-6.

33. Turmo-Garuz A, Rodas G, Balius R, et al. Can local corticosteroid injection in the retrocalcaneal bursa lead to rupture of the Achilles tendon and the medial head of the gastrocnemius muscle? Musculoskelet Surg. 2014:98:121-6.

34. Lohrer H. Results of reinsertion of the distal Achilles tendon. Z Orthop Unfall. 2019;157:246-53.

35. MacDermid JC, Silbernagel KG. Outcome evaluation in tendinopathy: foundations of assessment and a summary of selected measures. J Orthop Sports Phys Ther. 2015;45:950-64.

36. Mercieca-Bebber R, King MT, Calvert MJ, et al. The importance of patientreported outcomes in clinical trials and strategies for future optimization. Patient Relat Outcome Meas. 2018;9:353-67.

37. Kader D, Saxena A, Movin T, Maffulli N. Achilles tendinopathy: some aspects of basic science and clinical management. Br J Sports Med. 2002;36:239-49.

38. Lohrer H, Nauck T, Dorn NV, Konerding MA. Comparison of endoscopic and open resection for Haglund tuberosity in a cadaver study. Foot Ankle Int. 2006;27:445-50.

39. Filardo G, Presti ML, Kon E, Marcacci M. Nonoperative biological treatment approach for partial Achilles tendon lesion. Orthopedics. 2010;33:120-3.

40. Sarsilmaz A, Varer M, Coskun G, et al. Partial Achilles tendon rupture presenting with giant hematoma; MRI findings of 4 year follow up. Foot (Edinb ). 2011;21:204-6.

41. Medeiros DM. Conservative treatment of Achilles tendon partial tear in a futsal player: a case report. Physiother Theory Pract. 2019:1-8.

42. Huegel J, Boorman-Padgett JF, Nuss CA, et al. Quantitative comparison of three rat models of Achilles tendon injury: a multidisciplinary approach. J Biomech. 2019;88:194-200.

43. Khan KM, Forster BB, Robinson J, et al. Are ultrasound and magnetic resonance imaging of value in assessment of Achilles tendon disorders? A two year prospective study. Br J Sports Med. 2003;37:149-53.

\section{Publisher's Note}

Springer Nature remains neutral with regard to jurisdictional claims in published maps and institutional affiliations.

\section{Ready to submit your research? Choose BMC and benefit from:}

- fast, convenient online submission

- thorough peer review by experienced researchers in your field

- rapid publication on acceptance

- support for research data, including large and complex data types

- gold Open Access which fosters wider collaboration and increased citations

- maximum visibility for your research: over $100 \mathrm{M}$ website views per year

At BMC, research is always in progress.

Learn more biomedcentral.com/submissions 